\title{
THE FREE ENVELOPE OF A FINITELY GENERATED COMMUTATIVE SEMIGROUP
}

\author{
BY \\ PIERRE ANTOINE GRILLET
}

Let $S$ be a commutative semigroup. A quasi-universal free semigroup of $S$ is a free commutative semigroup with identity $F$ together with a homomorphism $\eta$ of $S$ into $F$ such that any homomorphism of $S$ into a free commutative semigroup with identity factors through $\eta$; if there is uniqueness in this factorization, we say that $(F, \eta)$ is a universal free semigroup of $S$.

If $S$ is finitely generated, there exists a "smallest" quasi-universal free semigroup of $S$; we call it the free envelope of $S$. Its construction and study is the first object of this paper, the second being the application of the free envelope to the study of cancellative and power-cancellative commutative semigroups.

We construct the free envelope in the first section. Cancellative and powercancellative semigroups appear in $\$ 2$; we prove that a finitely generated commutative semigroup is embeddable into a free commutative semigroup with identity if and only if it has these properties and has either no identity or a trivial group of units; then it is embeddable into its free envelope. The study of this latter embedding gives, conversely, a number of interesting properties of the free envelope in the general situation.

The dual of a finitely generated commutative semigroup $S$ may be defined as the semigroup $S^{*}$ of all homomorphisms of $S$ into the additive semigroup of all nonnegative integers, under pointwise addition. Using free envelopes, we prove in $\S 3$ that $S^{* * *} \cong S^{*}$ and investigate the relationship between $S$ and $S^{* *}$. This yields in turn a number of results concerning universal free semigroups when they exist. A study of various dimensions completes the section. $\$ 4$ deals with embeddings $S \subseteq T$ such that every relation which holds in $S$ can be deduced from the presentation of $S$ in $T$ without using any relation which may hold in $T$ (in which case we say that $T$ kills $S$ ). We show that, if $S$ is finitely generated, the (inclusion) homomorphism of $S$ into $T$ can be extended to the free envelope of $S$; if furthermore $T$ is cancellative, power-cancellative and without identity element, then $T$ contains subsemigroups which kill $S$ and are minimal with that property.

This paper has benefited from numerous suggestions, by the members of the Tulane semigroup seminar, especially William R. Nico and A. H. Clifford, and by our referee, which we acknowledge gladly.

Received by the editors December 9, 1968 and, in revised form, September 2, 1969.

Copyright (C) 1970, American Mathematical Society 
Throughout, "semigroup" will mean "commutative semigroup". All semigroups will be denoted additively; thus, what would be $S^{1}$ is denoted by $S^{0}$. The set of all positive integers (all integers, all rational numbers, all real numbers) is denoted $N(Z, Q, R)$. The free (commutative) semigroup on a set $X$ is denoted by $N_{X}$, the free semigroup with identity on $X$ by $N_{X}^{0}$, the free $K$-module on $X$ by $K_{X}$. For convenience, finitely generated is abbreviated as f.g. The reader is referred to [1] for the background on semigroups.

\section{Construction of the free envelope.}

1. This construction depends on some immediate results on orthogonality in free abelian groups, which we recall first. Let $X$ be a nonempty set. We consider that $N_{X}^{0} \subset Z_{X} \subset Q_{X} \subset R_{X}$. If $B \subseteq Q_{X}, \bar{B}$ denotes the subspace of $Q_{X}$ generated by $B$. The coordinates of $a \in \boldsymbol{Q}_{\mathrm{X}}$ will be denoted by $a_{x}$, so that $a=\sum_{x \in X} a_{x} x$.

In $\boldsymbol{R}_{X}$ we have the inner product $a \cdot b=\sum_{x \in X} a_{x} b_{x}$. For any subset $B$ of $\boldsymbol{Q}_{X}$, $B^{\perp}$ denotes the set of all elements of $Z_{X}$ which are orthogonal to all elements of $B$ (so that the orthogonal of $B$ in $Q_{X}$ is $\overline{B^{\perp}}$ ). The following properties are immediate:

Proposition 1.1. For any subset $B$ of $Z_{X}, B^{\perp}=\bar{B}^{\perp} \cap Z_{X}=\left(\bar{B} \cap Z_{X}\right)^{\perp}$ and $B^{\perp \perp}$ $=\bar{B} \cap Z_{X}$ is the pure subgroup of $Z_{X}$ generated by $B$, so that $B^{\perp \perp \perp}=B^{\perp}$.

Say that $a \in Z_{X}$ is positive in case $a \neq 0$ and $a_{x} \geqq 0$ for all $x \in X$ (i.e. $a \in N_{X}$ ), and strictly positive in case $a_{x}>0$ for all $x \in X$ (which never happens when $X$ is infinite). The following lemma will be used later.

LEMMA 1.2. Let $X$ be finite and $G$ be a subgroup of $Z_{X}$ such that $G \cap N_{X}=\varnothing$. Then $G^{\perp}$ is generated (as a subgroup) by its strictly positive elements.

Proof. Let $P$ be the positive cone of $\boldsymbol{R}_{X}$ and $S$ be the subspace of $\boldsymbol{R}_{X}$ generated by $G$. Now $\bar{G}$ contains no positive element, since such element could be multiplied by a positive integer to yield a positive element of $G$. It follows that $S$ contains no positive element either. The angles between vectors in $S$ and vectors in $P$ are then bounded below by some positive number, which permits to find a convex open subset $U$ of $\boldsymbol{R}_{X}$ containing $P$ and disjoint from $S$ (e.g. all vectors making a small enough angle with a vector in $P$ ). Then the Hahn-Banach Theorem yields a subspace $H$ of $\boldsymbol{R}_{X}$ containing $S$ and disjoint from $U$, hence containing no positive element, and of codimension one.

Let $a \neq 0$ be orthogonal to $H$ in $\boldsymbol{R}_{X}$, so that $u \in H$ if and only if $a \cdot u=0$. Since $H \cap P=\varnothing, a_{x} \neq 0$ for all $x \in X$; furthermore all the numbers $a_{x}$ have same sign, for $a_{x}>0$ and $a_{y}<0$ would imply $a_{x} y-a_{y} x \in H \cap P$. Hence either $a$ or $-a$ is strictly positive, and the orthogonal $K$ of $S$ in $\boldsymbol{R}_{X}$ contains a strictly positive element. Now $K$ is the solution space of a system of linear equations with rational coefficients; hence $K \cap Q_{X}$ is dense in $K$, so that $K$ contains a strictly positive element whose coordinates are rational. Multiplying this element by a suitable integer yields a strictly positive element of $G^{\perp}$. 
Finally, let $k$ be a strictly positive element of $G^{\perp}$. For any $u \in G^{\perp}$, set $M=\max \left|u_{x}\right|$; then $(M+1) k+u$ is strictly positive, so that $u$ is the difference of two strictly positive elements of $G^{\perp}$. Therefore $G^{\perp}$ is generated, as a subgroup of $Z_{X}$, by its strictly positive elements.

2. Now let $S$ be a f.g. (commutative) semigroup, and $X$ be a finite generating subset of $S$. (If $S$ has an identity, $X$ need generate $S$ as a semigroup with identity only.)

For each relation $r: \sum_{x \in X} r_{x}^{\prime} x=\sum_{x \in X} r_{x}^{\prime \prime} x$ between elements of $X$, set $\bar{r}=$ $\sum_{x \in X}\left(r_{x}^{\prime}-r_{x}^{\prime \prime}\right) x \in Z_{X}$. The Rédei group of $S$ (relative to $X$ ) is the set $R=\{\bar{r} ; r$ holds in $S$; ; it is indeed a subgroup of $Z_{X}$, for 0 can be obtained from the trivial relations, $-\bar{r}$ by exchanging the sides of $r$ and $\bar{r}+\bar{s}$ by just adding $r$ and $s$ formally. We let $K=R^{\perp}, C=K \cap N_{X}^{0}$ and $B$ be the set of all irreducible elements of $C$ (i.e. $b \in B$ if and only if $b \in C, b \neq 0$, and $b=c+d, c, d \in C$ implies $c=0$ or $d=0)$. Since $X$ is finite, Corollary 9.19 of [1] (a consequence of Wilson's Theorem) implies that $B$ is finite and generates $C$ (as a semigroup with identity).

Let $F$ be the free semigroup with identity on the set $B$ (if $B=\varnothing$, i.e. $C=\{0\}$, then $F=\{0\}$ is trivial). We have a canonical homomorphism of $S$ into $F$, defined as follows. For each $x \in X$, let $\alpha(x)=\sum_{b \in B} b_{x} b \in N_{B}^{0}=F$. Whenever $r: \sum_{x \in X} r_{x}^{\prime} x$ $=\sum_{x \in X} r_{x}^{\prime \prime} x$ holds in $S$, then $\sum_{x \in X} \bar{r}_{x} b_{x}=0$ for each $b \in B$, so that $\sum_{x \in X} r_{x}^{\prime} \alpha(x)$ $=\sum_{x \in X} r_{x}^{\prime \prime} \alpha(x)$ holds in $F$. Therefore $\alpha$ can be extended to a homomorphism of $S$ into $F$, which will also be denoted by $\alpha$, and is well defined by $\alpha\left(\sum_{x \in X} r_{x} x\right)$ $=\sum_{x \in X} r_{x} \alpha(x)$. The pair $(F, \alpha)$ is the free envelope of $S$; if $S$ or $X$ are not kept fixed, $F$ will be denoted by $F_{X}, F(S)$ or $F_{X}(S)$, and similarly for $R, K, C, B$.

Proposition 1.3. There is a canonical isomorphism $\Phi_{X}: C_{X} \cong \operatorname{Hom}\left(S, N^{0}\right)$. Hence the following are equivalent: (i) $F_{X}(S)$ is not trivial; (ii) there exists a nonzero homomorphism of $S$ into $N^{0}$; (iii) there exists a nonzero homomorphism of $S$ into some free semigroup with identity.

Proof. If $c \in C$, put $\Phi(c)(x)=c_{x} \in N^{0}$. Whenever $r: \sum_{x \in X} r_{x}^{\prime} x=\sum_{x \in X} r_{x}^{\prime \prime} x$ holds in $S, \sum_{x \in X} r_{x}^{\prime} c_{x}=\sum_{x \in X} r_{x}^{\prime \prime} c_{x}$ holds in $N^{0}$ since $\bar{r} \cdot c=0$; therefore $\Phi(c)$ can be extended to a homomorphism of $S$ into $N^{0}$, also denoted by $\Phi(c)$. If conversely $\varphi$ is a homomorphism of $S$ into $N^{0}$, the same argument shows that $\Gamma(\varphi)=\sum_{x \in X} \varphi(x) x \in Z_{X}$ lies in $C$. It is immediate to show that $\Gamma$ and $\Phi$ are inverse isomorphisms.

It follows that (i) and (ii) are equivalent. Clearly (ii) implies (iii); if conversely there exists a nonzero homomorphism of $S$ into a free semigroup $N_{Y}^{0}$ with identity, then its composition with the $y$ th projection is a nonzero homomorphism of $S$ into $N^{0}$ for some $y \in Y$.

Note that, if $F_{X}(S)$ is not trivial, then $\alpha$ is not zero.

THEOREM 1.4. Up to isomorphism, the free envelope of $S$ does not depend on the choice of $X$.

Proof. If $Y$ is another finite generating subset of $S$, we have to find an isomorphism 
$\theta: F_{X} \cong F_{Y}$ such that $\alpha_{X}=\theta \circ \alpha_{Y}$. By 1.3 we know that $C_{X} \cong C_{Y}$, so that there is a one-to-one mapping $\theta$ of $B_{X}$ onto $B_{Y}$, which can be described explicitly as follows. For each $b \in B_{X}, \theta(b)=\Phi_{Y}^{-1}\left(\Phi_{X}(b)\right)=\sum_{y \in Y} \Phi_{X}(b)(y) y$; since $X$ generates $S$, we may write $y=\sum_{x \in X} n_{x}^{y} x$ for each $y \in Y$, and then

$$
\theta(b)=\sum_{y \in Y} \sum_{x \in X} \Phi_{X}(b)(x) n_{x}^{y} y=\sum_{y \in Y} \sum_{x \in X} b_{x} n_{x}^{y} y \in Z_{Y}
$$

Let $\theta$ also denote the induced isomorphism $F_{X} \cong F_{Y}$. Then, for each $y \in Y$ :

$$
\theta\left(\alpha_{X}(y)\right)=\sum_{b \in B_{X}} \sum_{x \in X} n_{x}^{y} b_{x} \theta(b)=\sum_{\theta(b) \in B_{Y}} \theta(b)_{y} \theta(b)=\alpha_{Y}(y) .
$$

Since $Y$ generates $S$, it follows that $\theta \circ \alpha_{X}=\alpha_{Y}$.

3. THEOREM 1.5. Let $S$ be a finitely generated (commutative) semigroup. The free envelope of $S$ is a quasi-universal free semigroup of $S$.

Proof. Let $\varphi$ be a homomorphism of $S$ into a free semigroup $N_{Y}^{0}$ with identity and $X$ be a finite generating subset of $S$. For each $x \in X$, set $\varphi(x)=\sum_{y \in Y} m_{y}^{x} y$ (where $m_{y}^{x} \in N^{0}$ and $\left\{y \in Y ; m_{y}^{x} \neq 0\right\}$ is finite). If $r: \sum_{x \in X} r_{x}^{\prime} x=\sum_{x \in X} r_{x}^{\prime \prime} x$ holds in $S$, then $\sum_{x \in X} r_{x}^{\prime} \varphi(x)=\sum_{x \in X} r_{x}^{\prime \prime} \varphi(x)$ must hold in $N_{Y}^{0}$, i.e. $\bar{r} \cdot \sum_{x \in X} m_{y}^{x} x=0$ in $Z_{X}$ for all $y \in Y$. Therefore $\sum_{x \in X} m_{y}^{x} x \in C$ for each $y$ and there exist integers $n_{y}^{b} \geqq 0$ such that $\sum_{x \in X} m_{y}^{x} x=\sum_{b \in B} n_{y}^{b} b$ for each $y \in Y$. If $m_{y}^{x}=0$ for all $x$, we must take $n_{y}^{b}=0$ for all $b$, so that, for each $b \in B,\left\{y \in Y ; n_{y}^{b} \neq 0\right\}$ is finite. Therefore there exists a homomorphism $\psi$ of $F(S)$ into $N_{Y}^{0}$ such that $\psi(b)=\sum_{y \in Y} n_{y}^{b} y$ for all $b \in B$.

For each $x \in X, m_{y}^{x}=\sum_{b \in B} n_{y}^{b} b_{x}$, whence

$$
\psi(\alpha(x))=\sum_{y \in Y} \sum_{b \in B} n_{y}^{b} b_{x} y=\sum_{y \in Y} m_{y}^{x} y=\varphi(x) ;
$$

since $X$ generates $S$, it follows that $\psi \circ \alpha=\varphi$. Therefore $(F(S), \alpha)$ is a quasi-universal free semigroup of $S$.

Observe that we could not use McAlister's technique (cf. [5]) to construct a quasi-universal free semigroup of $S$ since the class of all free semigroups with identity is not implicational.

\section{Totally cancellative semigroups.}

1. We call a (commutative) semigroup totally cancellative (abbreviated as t.c.) if it is power-cancellative (i.e. $n a=n b$ implies $a=b$ for all $n \in N$; cf., e.g., [4]) and cancellative. A t.c. semigroup is reduced if either it has no identity element or its group of units is trivial.

LEMMA 2.1. Let $S$ be a t.c. semigroup having a finite generating subset $X$. Then $R_{X}(S)$ is a pure subgroup of $Z_{X}$, and a relation $r$ holds in $S$ if and only if $\bar{r} \in R_{X}(S)$. If furthermore $S$ is reduced, then $R_{X}(S)=B_{X}(S)^{\perp}$ (unless $\left.0 \in X\right)$. 
Proof. First, let $r: \sum_{x \in X} r_{x}^{\prime} x=\sum_{x \in X} r_{x}^{\prime \prime} x$ be such that $\bar{r} \in R$; so that $\bar{r}=\bar{s}$, where $s: \sum_{x \in X} s_{x}^{\prime} x=\sum_{x \in X} s_{x}^{\prime \prime} x$ holds in $S$. Since $r_{x}^{\prime}-r_{x}^{\prime \prime}=s_{x}^{\prime}-s_{x}^{\prime \prime}$ for all $x \in X$,

$$
\sum_{x \in X}\left(r_{x}^{\prime}+s_{x}^{\prime \prime}\right) x=\sum_{x \in X}\left(r_{x}^{\prime \prime}+s_{x}^{\prime}\right) x
$$

holds trivially in $S$, and $r$ holds, by cancellation. On the other hand, if $r$ holds in $S$, then $\bar{r} \in R$ trivially.

Assume now that $n u \in R$, where $u \in Z_{X}$ and $n \in N$. Put $r_{x}^{\prime}=u_{x}, r_{x}^{\prime \prime}=0$ when $u_{x} \geqq 0, r_{x}^{\prime}=0, r_{x}^{\prime \prime}=-u_{x}$ when $u_{x} \leqq 0$. Then $\bar{r}=u$ and, by the first part of the proof, $n\left(\sum_{x \in X} r_{x}^{\prime} x\right)=n\left(\sum_{x \in X} r_{x}^{\prime \prime} x\right)$ holds in $S$. Since $S$ is t.c., $r$ holds in $S$ and $u \in R$, which shows that $R$ is a pure subgroup of $Z_{X}$.

Finally, assume that $\sum_{x \in X} n_{x} x \in R \cap N_{X}$, so that $n_{x} \geqq 0$ for all $x$ and $n_{y}>0$ for some $y \in X$. Then, by the first part of the proof, $y=\left(n_{y}+1\right) y+\sum_{x \neq y} n_{x} x$ holds in $S$; then $S$ has an identity element, namely $0=\sum_{x \in X} n_{x} x \in S$, and either $S$ has a nontrivial unit or $0=y \in X$. Therefore, if $S$ is reduced and $0 \notin X$, then $R \cap N_{X}=\varnothing$. Then it follows from 1.2 that $K$ is generated, as a subgroup, by its strictly positive elements, and a fortiori by $C$; since $K$ is always pure, $K$ coincides with the pure subgroup generated by $C$, and, since $B$ generates $C$, with the pure subgroup generated by $B$. So $K=B^{\perp \perp}$. Since $R$ is pure, $R=R^{\perp \perp}=K^{\perp}=B^{\perp \perp}=B^{\perp}$.

THEOREM 2.2. Let $S$ be a finitely generated (commutative) semigroup. Then $S$ is embeddable into a free semigroup with identity if and only if $S$ is totally cancellative and reduced, and then $\alpha$ is one-to-one.

Proof. These conditions are obviously necessary. Assume, conversely, that $S$ is f.g., t.c. and reduced, and let $X$ be a finite generating subset of $S$; if $S$ has an identity, we assume also that $0 \notin X$. With the usual notation, let $s=\sum_{x \in X} m_{x} x$, $t=\sum_{x \in X} n_{x} x \in S$ be such that $\alpha(s)=\alpha(t)$. Then, for all $b \in B, \sum_{x \in X} m_{x} b_{x}=\sum_{x \in X} n_{x} b_{x}$, so that $\sum_{x \in X}\left(m_{x}-n_{x}\right) x \in B^{\perp}$. By 2.1 this implies that $s=t$ holds in $S$; thus $\alpha$ is one-to-one, and $S$ is embeddable into its free envelope.

Note that the existence of an embedding of $S$ into a free semigroup with identity implies that $\alpha$ is one-to-one, by 1.5 .

2. A first consequence of these results is a universal property for $\alpha(S)$ in the general case.

PROPOSITION 2.3. Every f.g. semigroup $S$ has a greatest t.c. reduced homomorphic image, namely $\alpha(S)$.

Proof. Clearly $\alpha(S)$ is t.c., reduced, and a homomorphic image of $S$. Let now $T$ be a t.c. reduced semigroup and $\varphi$ be a homomorphism of $S$ into $T$. Since $S$ is f.g., there exists a f.g. subsemigroup $U$ of $T$ such that $\varphi$ is a homomorphism of $S$ into $U$. Since $U$ is also t.c. and reduced, it can be embedded into a free semigroup with identity; then there exists a homomorphism $\psi$ of $F(S)$ into this free semigroup such that $\psi \circ \alpha=\varphi$. The restriction of $\psi$ to $\alpha(S)$ sends $\alpha(S)$ into $T$, which shows that $\varphi$ factors through $\alpha$. This factorization is unique since $\alpha$ is onto $\alpha(S)$. 
Observe that the existence of a greatest t.c. reduced homomorphic image of $S$ can be established directly. Indeed $S$ has a smallest t.c. congruence $\mathscr{C}$; it can be described by: $a \mathscr{C} b$ if and only if $n a+c=n b+c$ for some $n \in N, c \in S$. If the greatest t.c. homomorphic image $T=S / \mathscr{C}$ is not reduced, it has a nontrivial group of units $U$, and the greatest t.c. reduced homomorphic image of $S$ can be obtained by further dividing $T$ by the smallest t.c. congruence $\mathscr{R}$ which makes $U$ trivial; $\mathscr{R}$ can be described by: $a \mathscr{R} b$ if and only if $n a+u=n b$ for some $n \in N, u \in U$. Hence 2.3 may be interpreted as giving a description of the congruence induced by $\alpha$.

Our next result is that the free envelope of $S$ depends only on the greatest t.c. reduced homomorphic image of $S$. This, along with an elementary characterization of the free envelope, will result from certain properties of the embedding of $\alpha(S)$ in $F(S)$. For later convenience, we state these properties in terms of $T \subseteq N_{W}^{0}$ :

(i) if $z \in W$ is such that $t_{z}=\sum_{w \in W} n_{w} t_{w}$ for all $t \in T$, where $n_{w} \in N^{0}$, then $n_{z}=1$ and $n_{w}=0$ for all $w \neq z$;

(ii) if the integers $n_{w}$ are such that $\sum_{w \in W} n_{w} t_{w} \geqq 0$ for all $t \in T$, then there exist integers $m_{w} \geqq 0$ such that $\sum_{w \in W} n_{w} t_{w}=\sum_{w \in W} m_{w} t_{w}$ for all $t \in T$;

(iii) if $p \in N$ divides $\sum_{w \in W} n_{w} t_{w}$ for all $t \in T$, where $n_{w} \in N^{0}$, then there exist integers $m_{w} \geqq 0$ such that $\sum_{w \in W} n_{w} t_{w}=\sum_{w \in W} p m_{w} t_{w}$ for all $t \in T$.

Note that, if $Y$ generates $T$, one obtains equivalent conditions by replacing "for all $t \in T$ " by "for all $y \in Y$ " in the above.

LEMMA 2.4. If $S$ is a f.g. semigroup, then conditions (i), (ii) and (iii) hold for $\alpha(S) \subseteq F(S)$.

Proof. Let $X$ be a finite generating subset of $S$. If $c \in B$ is such that $\alpha(x)_{c}=$ $\sum_{b \in B} n_{b} \alpha(x)_{b}$ for all $x \in X$, then $c_{x}=\sum_{b \in B} n_{b} b_{x}$ for all $x \in X$, whence $c=\sum_{b \in B} n_{b} b$ in $Z_{X}$ and $n_{c}=1, n_{b}=0$ if $b \neq c$, since $c \in B$ is an irreducible element of $C$. Therefore (i) holds. If similarly $\sum_{b \in B} n_{b} \alpha(x)_{b} \geqq 0$ for all $x \in X$, where $n_{b} \in Z$, then $\sum_{b \in B} n_{b} b_{x} \geqq 0$ for all $x \in X$, so that $\sum_{b \in B} n_{b} b \in N_{X}^{0} \cap K=C$ and there exist $m_{b} \in N^{0}$ such that $\sum_{b \in B} n_{b} b=\sum_{b \in B} m_{b} b$ in $Z_{X}$. Then $\sum_{b \in B} n_{b} \alpha(x)_{b}=\sum_{b \in B} n_{b} b_{x}=\sum_{b \in B} m_{b} b_{x}=\sum_{b \in B} m_{b} \alpha(x)_{b}$ for all $x \in X$. Therefore (ii) holds. The verification of (iii) is similar, using the fact that $K$ is a pure subgroup of $Z_{X}$.

LEMma 2.5. Let $T$ be a f.g. subsemigroup of $N_{W}^{0}$ such that (i), (ii), (iii) hold. Then $N_{\mathrm{W}}^{0}$, together with the inclusion, is (up to isomorphism) the free envelope of $T$.

Proof. Let $X$ be a finite generating subset of $T$. Observe that $r: \sum_{x \in X} r_{x}^{\prime} x$ $=\sum_{x \in X} r_{x}^{\prime \prime} x$ holds in $T$ if and only if $\sum_{x \in X} r_{x}^{\prime} x_{w}=\sum_{x \in X} r_{x}^{\prime \prime} x_{w}$ for all $w \in W$; if we set $\bar{w}=\sum_{x \in X} x_{w} x \in Z_{X}, \bar{W}=\{\bar{w} ; w \in W\}$, it follows from 2.1 that $R=\bar{W}^{\perp}$; therefore $K$ is the pure subgroup of $Z_{X}$ generated by $\bar{W}$. Observe also that, if $w, z \in W$, $\bar{w}=\bar{z}$ implies $w=z$ by (i); similarly $\bar{w}=0$ is impossible. We shall prove that $\bar{W}=B$.

We already know that $\bar{W} \subseteq C$ and that $0 \notin \bar{W}$. Next, let $c \in C$. Since $K$ is the pure subgroup generated by $\bar{W}$, there exist $n_{w} \in Z, p \in N$ such that $p c=\sum_{w \in W} n_{w} \bar{w}$; since $\sum_{w \in W} n_{w} x_{w}=p c_{x} \geqq 0$ for all $x \in X$, it follows from (ii) that there exist integers 
$m_{w} \geqq 0$ such that $p c=\sum_{w \in W} m_{w} \bar{w}$; then $p$ divides $p c_{x}=\sum_{w \in W} m_{w} x_{w}$ for all $x \in X$, and by (iii) there exist integers $k_{w} \geqq 0$ such that $p c=\sum_{w \in W} m_{w} \bar{w}=\sum_{w \in W} p k_{w} \bar{w}$; finally $c=\sum_{w \in W} k_{w} \bar{w}$. Therefore $C$ is generated by $\bar{W}$. In particular $\bar{W}$ must contain all irreducible elements of $C$, i.e. $B \subseteq \bar{W}$. Conversely, every $\bar{z} \in \bar{W}$ is irreducible: for $\bar{z}=\sum_{w \in W} n_{w} \bar{w}$, where $n_{w} \in N^{0}$ and $\sum_{w \in W} n_{w} \geqq 2$, is impossible by (i). So $\bar{W}=B$.

Consequently there exists an isomorphism $\theta$ of $N_{W}^{0}$ onto $F(T)=N_{W}^{0}$ such that $\theta(w)=\bar{w}$ for all $w \in W$. If $\beta$ is the inclusion homomorphism of $T$ into $N_{W}^{0}$, then, for all $x \in X: \theta(x)=\sum_{w \in W} x_{w} \bar{w}=\alpha(x)$. It follows that $\theta \circ \beta=\alpha$, which completes the proof.

The following theorems are immediate consequences of these two lemmas.

THEOREM 2.6. Let $T$ be a finitely generated subsemigroup of a free (commutative) semigroup with identity $F$. Then $F$ is the free envelope of $T$ if and only if (i), (ii), (iii) hold.

THEOREM 2.7. Let $S$ be finitely generated. Up to isomorphism, $S$ and $\alpha(S)$ have same free envelope.

THEOREM 2.8. Let $S$ be finitely generated and $\varphi$ be a homomorphism of $S$ into a free semigroup with identity $F$. Then $(F, \varphi)$ is (up to isomorphism) the free envelope of $S$ if and only if (i), (ii), (iii) hold for $\varphi(S) \subseteq F$ and $\varphi$ induces the same congruence as $\alpha$.

3. Here are some applications of these results.

THEOREM 2.9. Let $(F(S), \alpha)$ be the free envelope of $S$. The only endomorphism of $F(S)$ which leaves every element of $\alpha(S)$ fixed is the identity.

Proof. Let $\eta$ be an endomorphism of $F$; for each $b \in B$, set $\eta(b)=\sum_{c \in B} n_{c}^{b} c$. If $\eta(u)=u$ for each $u \in \alpha(S)$, then $\sum_{b \in B} \sum_{c \in B} \alpha(x)_{b} n_{c}^{b} c=\sum_{c \in B} \alpha(x)_{c} c$ for all $x \in X$, whence, for each $c \in B, x \in X: \alpha(x)_{c}=\sum_{b \in B} n_{c}^{b} \alpha(x)_{b}$. Then it follows from (i) that $n_{c}^{c}=1$ and $n_{c}^{b}=0$ if $b \neq c$. Therefore $\eta$ is the identity.

THEOREM 2.10. Let $(F, \beta)$ be a quasi-universal free semigroup of $S$, where $S$ is finitely generated. There exists a one-to-one homomorphism $\mu$ of $F(S)$ into $F$ such that $\mu \circ \alpha=\beta$.

Proof. By 1.5 there exists a homomorphism $\mu$ of $F(S)$ into $F$ such that $\mu \circ \alpha=\beta$. Also there exists a homomorphism $\varphi$ of $F$ into $F(S)$ such that $\varphi \circ \beta=\alpha$. Then $\varphi \circ \mu$ is an endomorphism of $F(S)$ and leaves every element of $\alpha(S)$ fixed: it must be the identity, so that $\mu$ is one-to-one.

THEOREM 2.11. Let $S$ be a finitely generated (commutative) semigroup and $\beta$ be a homomorphism of $S$ into a free semigroup $F$ with identity. Then $(F, \beta)$ is (up to isomorphism) the free envelope of $S$ if and only if it is a quasi-universal free semigroup of $S$ and the only endomorphism of $F$ which leaves every element of $\beta(S)$ fixed is the identity. 
Proof. Continuing the proof of $2.10, \mu \circ \varphi$ is an endomorphism of $F$ which leaves every element of $\beta(S)$ fixed; hence it is also the identity, so that $\mu$ is an isomorphism. The converse is now trivial.

3. Duality and universal free semigroups: dimension.

1. The dual of a semigroup $S$ is here the semigroup $S^{*}=\operatorname{Hom}\left(S, N^{0}\right)$ under pointwise addition; it is a t.c. reduced semigroup with identity. In general we can iterate the process and obtain the bidual $S^{* *}$ of $S$; it comes with a canonical evaluation homomorphism $\sigma$ of $S$ into $S^{* *}$, defined by $\sigma(s)(\varphi)=\varphi(s)$ for all $s \in S$, $\varphi \in S^{*}$.

When $S$ is f.g. we can describe all this in terms of free envelopes, relative to any finite generating subset $X$. It has already been noted that $S^{*} \cong C_{X}(S)$, where the isomorphism is explicit. It follows that $S^{*}$ is finitely generated, by $\Phi(B)$ (where $\Phi(b)(x)=b_{x}$ for all $\left.x \in X\right)$. To simplify we shall identify $b$ and $\Phi(b)$, when $b \in B$ and $X$ is fixed.

Proposition 3.1. In $Z_{B}: R_{B}\left(S^{*}\right)=\alpha(S)^{\perp} ; K_{B}\left(S^{*}\right)$ is the pure subgroup generated by $\alpha(S) ; C_{B}\left(S^{*}\right) \cong S^{* *}$ is the intersection of $N_{B}^{0}=F(S)$ and the pure subgroup of $Z_{B}$ generated by $\alpha(S)$.

Proof. $r: \sum_{b \in B} r_{b}^{\prime} b=\sum_{b \in B} r_{b}^{\prime \prime} b$ holds in $S^{*}$ if and only if it holds in $C_{X}(S)$, if and only if $\sum_{b \in B}\left(r_{b}^{\prime}-r_{b}^{\prime \prime}\right) b_{x}=0$ for all $x \in X$, if and only if, in $Z_{B}, \bar{r} \cdot \alpha(x)=0$ for all $x \in X$, if and only if $\bar{r}$ is orthogonal to $\alpha(S)$. Therefore $R_{B}\left(S^{*}\right)=\alpha(S)^{\perp}$. The other assertions follow.

Let $\Phi^{*}$ be the canonical isomorphism $C_{B}\left(S^{*}\right) \cong S^{* *}$.

Proposition 3.2. $\Phi^{*} \circ \alpha=\sigma$.

Proof. By definition, $\Phi^{*}(c)(b)=c_{b}$ for all $c \in C_{B}\left(S^{*}\right), b \in B \subseteq S^{*}$. Thus, for all $x \in X, b \in B$ :

$$
\Phi^{*}(\alpha(x))(b)=\alpha(x)_{b}=b_{x}=\Phi(b)(x)=\sigma(x)(\Phi(b))=\sigma(x)(b) .
$$

Since $B$ generates $S^{*}, \Phi^{*}(\alpha(x))=\sigma(x)$ for all $x$, whence $\Phi^{*} \circ \alpha=\sigma$.

2. Here are some consequences of these results.

Proposition 3.3. If $S$ is f.g., $\sigma(S)$ is the greatest t.c. reduced homomorphic image of $S$.

Proof. This follows from 3.2 and 2.3.

Proposition 3.4. If $S$ is f.g., then $\sigma$ is one-to-one if and only if $S$ is t.c. and reduced.

Proof. This follows from 3.3 (or from 2.2).

Proposition 3.5. If $S$ is f.g., then $\sigma$ is an epimorphism relatively to t.c. semigroups.

Proof. This is to say that, if $\varphi, \psi$ are homomorphisms of $S^{* *}$ into a t.c. semigroup $T$ and if $\varphi \circ \sigma=\psi \circ \sigma$, then $\varphi=\psi$. By 3.2, it is enough to prove the same prop- 
erty for the homomorphism $\alpha$ of $S$ into $C_{B}\left(S^{*}\right)$; hence let $\varphi, \psi$ be homomorphisms of $C_{B}\left(S^{*}\right)$ into a t.c. semigroup $T$, such that $\varphi \circ \alpha=\psi \circ \alpha$. They induce homomorphisms $\bar{\varphi}, \psi$ of the universal group (=group of differences) $G$ of $C_{B}\left(S^{*}\right)$ into the universal group $G(T)$ of $T$. Since $T$ is t.c., $G(T)$ is a torsion free abelian group, so that $H=\operatorname{Ker}(\bar{\varphi}-\bar{\psi})$ is a pure subgroup of $G$; also $\alpha(S) \subseteq H$ since $\varphi \circ \alpha=\psi \circ \alpha$. On the other hand, $C_{B}\left(S^{*}\right) \subseteq Z_{B}$, so that we may take $G \subseteq Z_{B}$; since $S^{*}$ is t.c. and reduced, $K_{B}\left(S^{*}\right)$ is generated (as a subgroup) by $C_{B}\left(S^{*}\right)$ (see proof of 2.1), so that $G=K_{B}\left(S^{*}\right)$ and, by $3.1, G$ is the pure subgroup of $Z_{B}$ generated by $\alpha(S)$. Now, since $H$ is pure in $G$, it is also pure in $Z_{B}$; since $\alpha(S) \subseteq H$, this implies that $H=G$. Therefore $\bar{\varphi}-\bar{\psi}=0$, and $\varphi=\psi$.

Proposition 3.6. If $S$ is f.g., then $\sigma(S)$ is dense in $S^{* *}$ relative to t.c. semigroups.

Proof. This is to say that, if a homomorphism $\varphi$ of $S^{* *}$ into a t.c. semigroup $T$ is one-to-one on $\sigma(S)$, then it must be one-to-one. We use the same method and notation as in the previous proof. By 3.2 we may start from a homomorphism $\varphi$ of $C_{B}\left(S^{*}\right)$ into a t.c. semigroup $T$; $\varphi$ induces a homomorphism $\bar{\varphi}$ of $G$ into $G(T)$, whose kernel $H$ is a pure subgroup of $G$. Assume that $\varphi$ is one-to-one on $\alpha(S)$. For any $h \in H$, there exist, since $G$ is the pure subgroup of $Z_{B}$ generated by $\alpha(S)$, an integer $n>0$ and $u, v \in \alpha(S)$ such that $n h=u-v$; since $h \in \operatorname{Ker} \bar{\varphi}, \bar{\varphi}(u)=\bar{\varphi}(v)$ and the assumption on $\varphi$ implies $u=v$ and $h=0$. This proves that $H=0$. Therefore $\bar{\varphi}$ is one-to-one, and so is $\varphi$.

Proposition 3.7. Let $S$ be f.g. Up to isomorphism, $S$ and $S^{* *}$ have same free envelope.

Proof. It is enough to verify that the conditions (i), (ii), (iii) of $\$ 2$ hold for $C=C_{B}\left(S^{*}\right) \subseteq F(S)$; and we already know that these conditions are satisfied by $\alpha(S)$. Hence, if $c \in B$ is such that $u_{c}=\sum_{b \in B} n_{b} u_{b}$ for all $u \in C$, where $n_{b} \in N^{0}$, then this holds for all $u \in \alpha(S)$, whence $n_{c}=1$ and $n_{b}=0$ if $b \neq c$; therefore (i) holds. If similarly the integers $n_{b}$ are such that $\sum_{b \in B} n_{b} u_{b} \geqq 0$ for all $u \in C$, then this holds for all $u \in \alpha(S)$, so that there exist integers $m_{b} \geqq 0$ such that $\sum_{b \in B} n_{b} u_{b}=\sum_{b \in B} m_{b} u_{b}$ for all $u \in \alpha(S)$; by 3.1 every element of $C$ is a linear combination of elements of $\alpha(S)$, with coefficients in $Q$, and therefore the latter equality also holds for all $u \in C$. This proves (ii). The verification of (iii) is similar.

Proposition 3.8. If $S$ is f.g., then $S^{*} \cong S^{* * *}$.

Proof. The evaluation homomorphism $\tau$ of $S^{*}$ into $S^{* * *}$ (which is natural in $S$ ) will serve. First, if $\varphi$ is a homomorphism of $S$ into $N^{0}$, then $\tau(\varphi)(\sigma(s))=\sigma(s)(\varphi)=\varphi(s)$ for all $s \in S$, so that $\varphi=\tau(\varphi) \circ \sigma$. Hence, for any homomorphism $\psi$ of $S^{* *}$ into $N^{0}, \psi \circ \sigma=\tau(\psi \circ \sigma) \circ \sigma$; by 3.5 , this implies $\psi=\tau(\psi \circ \sigma)$, which shows that $\tau$ is onto. On the other hand, $\tau$ is one-to-one by 3.4 .

Incidentally, we have shown that the inverse isomorphism is the map $\sigma^{*}$ induced by $\sigma\left(\right.$ defined by $\left.\sigma^{*}(\psi)=\psi \circ \sigma\right)$. 
3. Our duality can in turn be applied to the study of universal free semigroups.

THEOREM 3.9. Let $S$ be a finitely generated (commutative) semigroup. The following conditions are equivalent:

(a) $F(S)$ is a universal free semigroup of $S$;

(b) there exists a universal free semigroup of $S$;

(c) $S^{*}$ is free;

(d) the pure subgroup of $Z_{B}$ generated by $\alpha(S)$ is $Z_{B}$ itself.

Under these conditions, $\left(S^{* *}, \sigma\right)$ is a universal free semigroup of $S$.

Proof. Trivially, (a) implies (b). If (b) holds, and if $F$ is a universal free semigroup of $S$, then the universal property yields an isomorphism $\operatorname{Hom}\left(S, N^{0}\right) \cong \operatorname{Hom}\left(F, N^{0}\right)$, so that $S^{*} \cong F^{*}$. This implies first that $F^{*}$ is finitely generated; since $F^{*}$ is a direct product of copies of $N^{0}$, one for each generator of $F$, this makes $F$ finitely generated. Therefore $F^{*}$ is free and so is $S^{*}$. Next, if (c) holds, then by $3.1 \alpha(S)^{\perp}=0$, so that $\alpha(S)^{\perp \perp}=Z_{B}$ and (d) holds. Finally, if (d) holds, then $C_{B}\left(S^{*}\right)=F(S)$, and in 1.5 the factorizations through $\alpha$ are unique, by 3.2 and 3.5 , so that $(F(S), \alpha)$ is a universal free semigroup of $S$. It follows also from 3.2 that $\left(S^{* *}, \sigma\right)$ is then a universal free semigroup of $S$, too.

COROLlaRY 3.10. If $S$ is finitely generated and $(F, \beta)$ is a universal free semigroup of $S$, then $\beta$ is an epimorphism relative to t.c. semigroups and $\beta(S)$ is dense in $F$ relative to t.c. semigroups.

Proof. This follows from 3.9 and 3.5, 3.6.

In general, $\alpha$ is not an epimorphism relative to t.c. semigroups, but it is easy to show, by the same techniques as for 3.5, that in the category of t.c. semigroups the dominion [3] of $\alpha(S)$ in $F(S)$ is precisely $C_{B}\left(S^{*}\right)$.

Finally, there is a characterization of universal free semigroups similar to 2.6. This involves the following condition, where $T$ is a subsemigroup of $N_{W}^{0}$ :

(i') if $\sum_{w \in W} n_{w} t_{w}=0$ for all $t \in T$, where $n_{w} \in Z$, then $n_{w}=0$ for all $w$.

This says that the functions $t \mapsto t_{w}(w \in W)$ are linearly independent over $Z$ and implies condition (i) of $\S 2$.

THEOREM 3.11. Let $T$ be a finitely generated subsemigroup of a free (commutative) semigroup $F=N_{W}^{0}$ with identity. Then $F$, together with the inclusion, is a universal free semigroup of $T$ if and only if (i'), (ii), (iii) hold.

Proof. If $F$ is a universal free semigroup of $T$, then it is also, up to isomorphism, the free envelope of $T$, so that conditions (i), (ii), (iii) of $\S 2$ hold. It remains to prove (i'). Relative to a finite generating subset $X$ of $T$, the proof of 2.5 shows that $B_{X}(T)$ is the set of all $\bar{w}=\sum_{x \in X} x_{w} x \in Z_{X}$. Since $T$ has a universal free semigroup, $C_{X}(T) \cong T^{*}$ is free, hence free on $B_{X}(T)$, so that there can be no nontrivial relation between the $\bar{w}$ 's in $Z_{X}$. Therefore, if the integers $n_{w}$ are such that $\sum_{w \in W} n_{w} t_{w}=0$ for all $t \in T$, then $\sum_{w \in W} n_{w} \bar{w}=0$ in $Z_{X}$, which implies $n_{w}=0$ for all $w$. This proves (i'). 
Conversely, assume that (i'), (ii), (iii) hold. Since (i') implies (i), $F$ “is" again the free envelope of $T$ and we may use the same material from the proof of 2.5 . With the same notation, if the integers $n_{w}$ are such that $\sum_{w \in W} n_{w} \bar{w}=0$, then $\sum_{w \in W} n_{w} t_{w}=0$ for all $t \in X$, hence also for all $t \in T$, so that $n_{w}=0$ for all $w$. It follows that no nontrivial relation holds in $Z_{X}$ between the $\bar{w}$ 's, so that $C_{X}(T)$ is free on $B_{X}(T)$. Since $T^{*}$ is free, the free envelope of $T$ is a universal free semigroup of $T$, and so is $F$. This completes the proof.

4. Let $S$ be t.c. Call a subset $Y$ of $S$ free if the subsemigroup of $S$ generated by $Y$ is free (commutative) over $Y$. The free dimension free $\operatorname{dim} S$ of $S$ is either the largest integer of the form Card $Y$, where $Y$ is a free subset of $S$, or infinite. If furthermore $S$ is f.g., we shall show that free $\operatorname{dim} S$ is finite and does not exceed the number of elements of any generating subset of $S$.

More precisely, let $X$ be a finite generating subset of $S$. The relational dimension of $S$ over $X$ is the rank rel $\operatorname{dim}_{X} S$ of $R_{X}(S)$, or, equivalently, the dimension of $\bar{R}_{X}(S)$ over $Q$. It can be interpreted as the largest number of independent defining relations, where independent means that no trivial relation can be obtained by finitely many formal additions or exchange of sides.

THEOREM 3.12. Let $S$ be a finitely generated cancellative (commutative) semigroup. For any finite generating subset $X$ of $S$, Card $X=$ free $\operatorname{dim} S+\operatorname{rel} \operatorname{dim}_{X} S$.

Proof. Let $Y$ be a free subset of $S$; for each $y \in Y$, write $y=\sum_{x \in X} n_{x}^{y} x$. If the integers $p_{y}$ are such that $\sum_{x \in X} \sum_{y \in Y} p_{y} n_{x}^{y} x \in R=R_{X}(S)$, then, writing $p_{y}=p_{y}^{\prime}-p_{y}^{\prime \prime}$, with $p_{y}^{\prime}, p_{y}^{\prime \prime} \in N^{0}$, the relation $r: \sum_{x \in X}\left(\sum_{y \in Y} p_{y}^{\prime} n_{x}^{y}\right) x=\sum_{x \in X}\left(\sum_{y \in Y} p_{y}^{\prime \prime} n_{x}^{y}\right) x$ is such that $\bar{r} \in R$; by $2.1, r$ holds in $S$ (this part of 2.1 does not use power-cancellativity). Hence $\sum_{y \in Y} p_{y}^{\prime} y=\sum_{y \in Y} p_{y}^{\prime \prime} y$, which implies $p_{y}=0$ for all $y$ since $Y$ is free.

Let $L$ be the linear mapping of $\boldsymbol{Q}_{Y}$ into $\boldsymbol{Q}_{X}$ such that $L(y)=\sum_{x \in X} n_{x}^{y} x$; it follows from the above that $L^{-1}(\bar{R})=0$. This shows, first, that $L$ is one-to-one (so that $Y$ is finite). Next $\boldsymbol{Q}_{Y} \cong L\left(\boldsymbol{Q}_{Y}\right)$ and $L\left(\boldsymbol{Q}_{Y}\right) \cap \bar{R}=0$. Taking dimensions over $\boldsymbol{Q}$, this yields

$$
\text { Card } Y=\operatorname{dim} L\left(Q_{Y}\right) \leqq \operatorname{dim} Q_{X}-\operatorname{dim} \bar{R}=\text { Card } X-\operatorname{rel} \operatorname{dim}_{X} S .
$$

Thus free $\operatorname{dim} S \leqq$ Card $X$-rel $\operatorname{dim}_{X} S$.

To prove the converse inequality, let $Y$ be a maximal free subset of $X$. For each $x \in X-Y, Y \cup\{x\}$ is not free, so that we can select a nontrivial relation $s^{x}$ between the elements of $Y \cup\{x\}$ which holds in $S$; note that the coefficient of $x$ in $\bar{s}^{x}$ is not zero, since $Y$ is free and $S$ is cancellative. It is then clear that $\left\{\bar{s}^{x} ; x \in X-Y\right\}$ is a linearly independent subset of $\bar{R}$, so that $\operatorname{rel} \operatorname{dim}_{X} S \geqq$ Card $X-\operatorname{Card} Y$. It follows that

$$
\text { free } \operatorname{dim} S \geqq \text { Card } Y \geqq \text { Card } X-\operatorname{rel} \operatorname{dim}_{X} S,
$$

which completes the proof.

Incidentally we proved that a free subset with maximal cardinality can be found among the subsets of $X$. 
Now define the generating dimension gen $\operatorname{dim} S$ of $S$ as the smallest number of the form Card $X$, where $X$ is a finite generating subset of $S$, and the relational dimension rel $\operatorname{dim} S$ as the smallest number of the form $\operatorname{rel} \operatorname{dim}_{X} S$. From 3.12 follows that gen $\operatorname{dim} S=$ free $\operatorname{dim} S+$ rel $\operatorname{dim} S$. If $S$ is t.c. and reduced, the following result shows that gen $\operatorname{dim} S$ is just the number of irreducible elements of $S$.

Proposition 3.13. Let $S$ be a f.g., t.c. reduced semigroup. Then $S$ is generated by its irreducible elements.

Proof. Since $S$ is embeddable into a free semigroup with identity by 2.2 , any element of $S$ has only finitely many divisors, and $s+t=s$ implies $t=0$ (and is impossible if $S$ has no identity element). Assume that $a \in S$ is not a sum of irreducible elements. Then $a$ is not irreducible, so that it can be written under the form $b+c$ (with $b, c \neq 0$ if $S$ has an identity element); then one of $b, c$ is not irreducible, so that $a$ can be written as a sum of three (nonzero) elements. Continuing thus, we see that for all $n \in N$ we can write $a$ as a sum $a=a_{1}+a_{2}+\cdots+a_{n}$ of $n$ (nonzero) elements. Taking $n$ larger than the number of divisors of $a$, we obtain $n$ distinct divisors of $a$, namely $a_{1}, a_{1}+a_{2}, \ldots, a_{1}+a_{2}+\cdots+a_{n}$, a contradiction. Therefore $S$ is generated by its irreducible elements.

Our last result concerns duality.

Proposition 3.14. If $S$ is f.g., t.c. and reduced, then free $\operatorname{dim} S^{*}=$ free $\operatorname{dim} S$ and $\operatorname{gen} \operatorname{dim} F(S)=$ free $\operatorname{dim} S+\operatorname{rel} \operatorname{dim} S^{*}$.

Proof. Relative to any finite generating subset $X$ of $S, K$ is the subgroup of $Z_{X}$ generated by $C$ and therefore

free $\operatorname{dim} S^{*}=$ free $\operatorname{dim} C=\operatorname{dim} \bar{K}=$ Card $X-\operatorname{dim} \bar{R}=$ free $\operatorname{dim} S$.

The second formula follows since gen $\operatorname{dim} F(S)=\operatorname{gen} \operatorname{dim} S^{*}$.

4. Killing.

1. Let $T$ be a (commutative) semigroup, $S$ be a subsemigroup of $T$ and $X, Y$ be generating subsets of $S, T$. Since $Y$ generates $T$, we can choose for each $x \in X$ a relation $p^{x}: x=\sum_{y \in Y} p_{y}^{x} y$ which holds in $T$. The family $p=\left(p^{x}\right)_{x \in X}$ is a presentation of $S \subseteq T$ relative to $X$ and $Y$. If $T$ is itself a subsemigroup of some semigroup $U$ generated by $Z$, and if $q$ is a presentation of $T \subseteq U$ relative to $Y$ and $Z$, so that $y=\sum_{z \in Z} q_{z}^{y} z$ holds in $U$ for all $y$, then $x=\sum_{z \in Z}\left(\sum_{y \in Y} p_{y}^{x} q_{z}^{y}\right) z$ holds in $U$ for all $x \in X$, which yields a presentation $p \circ q$ of $S \subseteq U$ relative to $X$ and $Z$.

If $p$ is a presentation of $S \subseteq T$ relative to $X$ and $Y$, then the relations of $S$ are consequences of the presentation and the relations of $T$. More precisely, each relation $r: \sum_{x \in X} r_{x}^{\prime} x=\sum_{x \in X} r_{x}^{\prime \prime} x$ between elements of $X$ induces a relation $r * p: \sum_{y \in Y}\left(\sum_{x \in X} r_{x}^{\prime} p_{y}^{x}\right) y=\sum_{y \in Y}\left(\sum_{x \in X} r_{x}^{\prime \prime} p_{y}^{x}\right) y$ between elements of $Y$; clearly $r$ holds in $S$ if and only if $r * p$ holds in $T$. One sees that, if $r$ is trivial, then so is $r * p$; and that $r *(p \circ q)=(r * p) * q$. 
We say that $p$ is a killing presentation in case $r * p$ is trivial whenever $r$ holds in $S$; in other words, if the relations of $S$ are consequences of $p$ alone, and not of the relations of $T$. We say that $T$ kills the relations of $S$ or, shortly, that $T$ kills $S$, if there exists a killing presentation of $S \subseteq T$ relative to some $X, Y$. (For instance, a free semigroup kills all its subsemigroups.)

Proposition 4.1. Let $S$ be a subsemigroup of $T$ and $X, X^{\prime}\left(Y, Y^{\prime}\right)$ be generating subsets of $S(T)$. If there exists a killing presentation of $S \subseteq T$ relative to $X$ and $Y$, then there exists a killing presentation of $S \subseteq T$ relative to $X^{\prime}$ and $Y^{\prime}$.

Proof. Let $p$ be a killing presentation of $S \subseteq T$ relative to $X$ and $Y$; take presentations $s$ of $S \subseteq S$ relative to $X^{\prime}$ and $X$ and $t$ of $T \subseteq T$ relative to $Y$ and $Y^{\prime}$; then $s \circ p \circ t$ is a presentation of $S \subseteq T$ relative to $X^{\prime}$ and $Y^{\prime}$. If furthermore a relation $r$ between elements of $X^{\prime}$ holds in $S$, then $r * s$ holds in $S$, so that $(r * s) * p$ is trivial; hence $r *(s \circ p \circ t)=((r * s) * p) * t$ is trivial. Therefore $s \circ p \circ t$ is a killing presentation.

Although 4.1 does not mean that, if $T$ kills $S$, then every presentation of $S \subseteq T$ is killing, it does say that killing is an intrinsic property of $S \subseteq T$, independent on the generating subsets chosen to express it. We shall in fact obtain an intrinsic characterization of killing (see 4.5 below), in the finitely generated case. In general, we have the following results.

Proposition 4.2. If $S \subseteq T \subseteq U$ and $T$ kills $S$, then $U$ kills $S$.

Proof. Relative to suitable generating subsets, let $p$ be a killing presentation of $S \subseteq T$ and $q$ be a presentation of $T \subseteq U$, such that $p \circ q$ is defined. Then $p \circ q$ is a killing presentation of $S \subseteq U$ : indeed, whenever $r$ holds in $S, r * p$ is trivial and so is $r *(p \circ q)=(r * p) * q$.

Proposition 4.3. If $S \subseteq T$, then $T$ kills $S$ if and only if $T^{0}$ kills $S$.

Proof. If $T$ kills $S$, then $T^{0}$ kills $S$ by 4.2. If conversely there exists a killing presentation of $S \subseteq T^{0}$ relative to $X$ and $Y$, say $p$, and if $T=T^{0}$, or $0 \notin Y$, then $p$ is a killing presentation of $S \subseteq T$. If $T \neq T^{0}$ and $0 \in Y$, then $Z=Y-\{0\}$ generates $T^{0}$ (as a semigroup with identity) and $q=\left(q^{x}\right)_{x \in X}, q^{x}: x=\sum_{z \in Z} p_{z}^{x} z$, is a presentation of $S \subseteq T$. It is immediate that $q$ is a killing presentation.

Proposition 4.4. If $T$ kills $S$ and $\varphi$ is a homomorphism of $T$ onto $U$ which is one-to-one on $S$, then $U$ kills $\varphi(S)$.

Proof. Let $p$ be a killing presentation of $S \subseteq T$ relative to $X$ and $Y$. Since $\varphi$ is onto, $\varphi(Y)$ generates $U$; we have a presentation $\varphi(p)$ of $\varphi(S) \subseteq U$ relative to $\varphi(X)$ and $\varphi(Y)$, defined by $\varphi(p)^{\varphi(x)}: \varphi(x)=\sum_{y \in Y} p_{y}^{x} \varphi(y)$. Relations can similarly be carried along $\varphi$. Now let $s$ be a relation between elements of $\varphi(X)$, which holds in $\varphi(S)$. Since $S \cong \varphi(S)$, we have $s=\varphi(r)$, where $r$ holds in $S$. Therefore $r * p$ is trivial, and so is $s * \varphi(p)=\varphi(r * p)$. This proves that $\varphi(p)$ is a killing presentation. 
Since any semigroup is a homomorphic image of a free semigroup, it will follow from 4.6 that the assumption that $\varphi$ is one-to-one on $S$ cannot be removed in 4.4.

2. The following result is fundamental.

THEOREM 4.5. Let $S$ be a finitely generated subsemigroup of a (commutative) semigroup T. Then $T$ kills $S$ if and only if there exists a homomorphism $\varphi$ of $F(S)$ into $T^{0}$ such that $\varphi \circ \alpha$ is the identity on $S$.

Proof. If such homomorphism $\varphi$ exists, then it is one-to-one on $\alpha(S)$; therefore $\varphi(F(S))$ kills $S$ by $4.4, T^{0}$ kills $S$ by 4.2 and $T$ kills $S$ by 4.3 .

Conversely, let $p$ be a killing presentation of $S \subseteq T$ relative to $X$ and $Y$, where $X$ is a finite generating subset of $S$. For each $y \in Y$, set $\bar{y}=\sum_{x \in X} p_{y}^{x} x \in Z_{X}$. Whenever $r: \sum_{x \in X} r_{x}^{\prime} x=\sum_{x \in X} r_{x}^{\prime \prime} x$ holds in $S, r * p: \sum_{y \in Y}\left(\sum_{x \in X} r_{x}^{\prime} p_{y}^{x}\right) y=\sum_{y \in Y}\left(\sum_{x \in X} r_{x}^{\prime \prime} p_{y}^{x}\right) y$ is trivial, which means that $\bar{r} \cdot \bar{y}=0$ in $Z_{X}$ for all $y \in Y$. Hence each $\bar{y}$ is in $C$ and there exist integers $m_{b}^{y} \geqq 0$ such that $\bar{y}=\sum_{b \in B} m_{b}^{y} b$. Then also $p_{y}^{x}=\sum_{b \in B} m_{b}^{y} b_{x}$.

For each $x \in X,\left\{y \in Y ; p_{y}^{x} \neq 0\right\}$ is finite; it follows that $\{y \in Y ; \bar{y} \neq 0\}$ is finite, so that $\left\{y \in Y ; m_{b}^{y} \neq 0\right\}$ is finite for each $b \in B$. Therefore there exists a homomorphism $\varphi$ of $F(S)$ into $T^{0}$ such that $\varphi(b)=\sum_{y \in Y} m_{b}^{y} y$ for all $b \in B$. If $x \in X, \varphi(\alpha(x))$ $=\sum_{y \in Y} \sum_{b \in B} b_{x} m_{b}^{y} b=\sum_{y \in Y} p_{y}^{x} y=x$; since $X$ generates $S, \varphi \circ \alpha$ is the identity on $S$, which completes the proof.

COROLlary 4.6. If $S$ is f.g., then $S$ is killed by some semigroup if and only if it is t.c. and reduced.

Proof. This follows from 2.2 and the theorem. (The only if part can be proved even when $S$ is not f.g., by noting that a killing presentation $p$ of $S \subseteq T$ relative to $X$ and $Y$ induces an embedding $\mu$ of $S$ into $N_{Y}^{0} ; \mu$ is defined by $\mu(x)=\sum_{y \in Y} p_{y}^{x} y \in N_{Y}^{0}$.)

COROLlary 4.7. A f.g. semigroup $S$ kills itself if and only if it is free.

Proof. If $S$ kills itself, then there exists a homomorphism $\varphi$ of $F(S)$ into $S^{0}$ such that $\varphi \circ \alpha$ is the identity on $S$. Then $\alpha \circ \varphi$ is an idempotent endomorphism of $F(S)$ and $(\alpha \circ \varphi)(F(S)) \cong S^{0}$. Therefore $S^{0}$ is a projective (commutative) semigroup with identity, and all such semigroups are free (cf., for instance, [2]). The converse is trivial.

COROLlaRY 4.8. If $S$ is f.g., then no proper subsemigroup of $F(S)$ kills $\alpha(S)$, except perhaps $F(S)-\{0\}$.

Proof. Let $T$ be a subsemigroup of $F(S)$ which kills $\alpha(S)$. Since $F(S)$ is also the free envelope of $\alpha(S)$ there exists a homomorphism $\varphi$ of $F(S)$ into $T^{0}$ which leaves every element of $\alpha(S)$ fixed. By 2.9, $\varphi$ must be the identity so that $T^{0}=F(S)$.

It is clear that $F(S)-\{0\}$ kills $\alpha(S)$ if and only if $0 \notin \alpha(S)$.

Finally, observe that 1.5 also follows from 4.5 .

3. The most important consequence of 4.5 is that any semigroup which kills $S$ must contain a subsemigroup which kills $S$ and can be completely described in 
terms of $F(S)$. More precisely, call canonical killer of $S$ any semigroup $T$ such that: (a) $S$ is a subsemigroup of $T$; (b) there exists a homomorphism $\varphi$ of $F(S)$ onto $T^{0}$ such that $\varphi \circ \alpha$ is the identity on $S$. Then 4.5 may be rephrased thus: $T$ kills $S$ if and only if $T^{0}$ contains a canonical killer of $S$.

The next question is, of course: does there exist minimal killers of $S$ (=such that no proper subsemigroup kills $S$ ) and does $T$ kill $S$ if and only if it contains a minimal killer of $S$ ?

We know by 4.8 that there exist minimal killers and by 4.5 that they must be canonical. There is also one case when our question has a very satisfactory answer, as far as only t.c. killers are concerned.

Proposition 4.9. If $S$ is f.g. and has a universal free semigroup $F$, then, up to isomorphism, there is only one t.c. minimal killer (namely, either $F$ or $F-\{0\}$ ) and a t.c. semigroup $T$ kills $S$ if and only if it contains it.

Proof. Up to isomorphism, $F=F(S)$, so that either $F$ or $F-\{0\}$ is a minimal killer of $S$. It follows from 3.10 that there is no other t.c. canonical killer, so that one has only one t.c. minimal killer (up to isomorphism). The result follows.

We are now going to show that 4.9 holds whenever $S$ is f.g., except for the uniqueness.

LEMMA 4.10. Let $S$ be f.g. and $T$ be t.c. reduced and a canonical killer of $S$. Then there are only finitely many canonical killers of $S$ in $T$.

Proof. Since $T$ is a canonical killer of $S$ there exists a homomorphism $\varphi$ of $F(S)$ onto $T^{0}$ such that $\varphi \circ \alpha$ is the identity on $S$. If a subsemigroup $U$ of $T$ is a canonical killer of $S$, there exists a homomorphism $\psi$ of $F(S)$ into $T^{0}$ such that $\psi \circ \alpha$ is the identity on $S$ (then $U^{0}=\psi(F(S))$ ). Therefore it is enough to prove that there are only finitely many such homomorphisms of $F(S)$ into $T^{0}$.

Now the congruence $\mathscr{C}$ induced by $\varphi$ is a cancellative congruence on $F(S)$; hence it follows from Redei's results (cf. [1], [6]) that $\mathscr{C}$ is determined by a subgroup $H$ of $Z_{B}$ by: $u \mathscr{C} v$ if and only if $u-v \in H$. Since $\mathscr{C}$ is also power-cancellative, $H$ is pure. Finally we claim that $G=H \cap N_{B}^{0}$ is the (free) subsemigroup of $N_{B}^{0}$ $=F(S)$ generated by $B^{\prime}=G \cap B$ (if $B^{\prime}=\varnothing$, then $G=\{0\}$ ). Indeed $u, v \in N_{B}^{0}$ and $u+v \in G$ implies $\varphi(u)+\varphi(v)=0$ in $T^{0}, \varphi(u)=\varphi(v)=0$ since $T^{0}$ is reduced and $u, v \in G$; if therefore $u=\sum_{b \in B} u_{b} b \in G$, then $b \in G$ whenever $u_{b}>0$, so that $u$ is a sum of elements of $B^{\prime}$.

Let $H^{\prime}=H \cap Z_{B^{\prime}}, H^{\prime \prime}=H \cap Z_{B^{\prime \prime}}$, where $B^{\prime \prime}=B-B^{\prime}$ and we consider that $Z_{B^{\prime}} \subseteq Z_{B}, Z_{B^{\prime \prime}} \subseteq Z_{B^{\prime}}$. By the above, $H^{\prime}=Z_{B^{\prime}}$ and $H^{\prime \prime} \cap N_{B^{\prime \prime}}=\varnothing$ since $H \cap N_{B}=N_{B^{\prime}}$. Furthermore $H=H^{\prime} \oplus H^{\prime \prime}$ : for if $u=\sum_{b \in B} u_{b} b \in H$, then $\sum_{b \in B^{\prime}} u_{b} b \in H^{\prime}$ and therefore $\sum_{b \in B^{\prime \prime}} u_{b} b \in H^{\prime \prime}$. Since $H$ is pure, so is $H^{\prime \prime}$. We shall now complete the description of $H$ by giving its equations in $Z_{B}$. Since $H^{\prime \prime}$ is pure in $Z_{B^{\prime \prime}}$, we have $H^{\prime \prime}=H^{\prime \prime \perp}$. Since $H^{\prime \prime} \cap N_{B^{n}}=\varnothing$, it follows from 1.2 that $H^{\prime \prime \perp}$ is generated by its strictly positive elements; thus we can find a finite subset $\left\{q^{i}=\sum_{b \in B^{n}} q_{b}^{i} b ; 1 \leqq i \leqq k\right\}$ which generates 
$H^{\prime \prime \perp}$ and satisfies $q_{b}^{i}>0$ for all $i$ and all $b \in B^{\prime \prime}$; then $H^{\prime \prime}$ is the orthogonal of that subset, so that $u=\sum_{b \in B^{\prime \prime}} u_{b} b \in H^{\prime \prime}$ if and only if $\sum_{b \in B^{\prime \prime}} q_{b}^{i} u_{b}=0$ for $1 \leqq i \leqq k$. It is now clear that $u=\sum_{b \in B} u_{b} b \in H$ if and only if $\sum_{b \in B^{\prime \prime}} q_{b}^{i} u_{b}=0$ for $1 \leqq i \leqq k$.

We are now equipped to find all homomorphisms $\psi$ of $F(S)$ into $T^{0}$ such that $\psi \circ \alpha$ is the identity on $S$. Since $\varphi(b)=0$ whenever $b \in B^{\prime}, T^{0}$ is generated by $\varphi\left(B^{\prime \prime}\right)$ and a homomorphism $\psi$ of $F(S)$ into $T^{0}$ is completely determined by any family $\left(n_{b}^{c}\right)_{c \in B, b \in B^{\prime \prime}}$ of nonnegative integers such that $\psi(c)=\sum_{b \in B^{\prime \prime}} n_{b}^{c} \varphi(b)$ for all $c \in B$. Furthermore, since $\varphi \circ \alpha$ is the identity on $S$, we have, relative to any finite generating subset $X$ of $S$, a presentation $x=\sum_{b \in B^{\prime \prime}} b_{x} \varphi(b)$ of $S \subseteq T^{0}$; whence $\psi \circ \alpha$ is the identity on $S$ if and only if $\sum_{b \in B^{\prime \prime}} b_{x} \varphi(b)=\sum_{b \in B^{\prime \prime}} \sum_{c \in B} c_{x} n_{b}^{c} \varphi(b)$ holds in $T^{0}$ for all $x \in X$, which means that $\sum_{b \in B^{\prime \prime}}\left(b_{x}-\sum_{c \in B} c_{x} n_{b}^{c}\right) b \in H$ for all $x \in X$. This is in turn equivalent to the system of equations:

$$
\sum_{b \in B^{\prime \prime}} \sum_{c \in B} q_{b}^{i} c_{x} n_{b}^{c}=\sum_{b \in B^{\prime \prime}} q_{b}^{i} b_{x} \quad(x \in X, 1 \leqq i \leqq k) .
$$

It now suffices to show that (1) has only finitely many solutions in nonnegative integers. Pick $b \in B^{\prime \prime}, c \in B$. Since $c \neq 0$, then $c_{y}>0$ for some $y \in X$. Then $q_{b}^{i} c_{y}>0$, so that $n_{b}^{c}$ may take only finitely many values in the solutions of the system. Since $B$ is finite, this completes the proof.

Note that any system of equations of $H$ leads to a system similar to (1) which may be used to find all the canonical killers of $S$ in $T$. In particular, one has a criterion for recognizing the minimal killers of $S$ that are t.c. and reduced.

THEOREM 4.11. Let $S$ be a finitely generated subsemigroup of a totally cancellative and reduced (commutative) semigroup $T$. Then $T$ kills $S$ if and only if it contains a minimal killer of $S$.

Proof. If $T$ kills $S$, then $T$ contains a canonical killer $U$ of $S$. Since $T$ is t.c. and reduced, so is $U$, and it follows from the lemma that $U$ contains a subsemigroup which is a canonical killer of $S$ and is minimal with this property. This subsemigroup is then a minimal killer of $S$. Thus $T$ contains a minimal killer of $S$. The converse is trivial.

5. Counterexamples and open questions.

1. The counterexamples give negative answers to the following questions concerning f.g., t.c. reduced semigroups:

5.1. If $S^{*}$ is free, need $S$ be free?

5.2. Is $S^{*}$ always free?

5.3. Is $\alpha(S)$ always dense in $F(S)$ relative to t.c. semigroups?

5.4. Are the minimal killers of $S$ always free? More precisely, does every minimal killer $T$ of $S$ satisfy

5.5. rel $\operatorname{dim} T<\operatorname{rel} \operatorname{dim} S$ ?

5.6. free $\operatorname{dim} T=$ free $\operatorname{dim} S$ ? (Note: it is clear that free $\operatorname{dim} S \leqq$ free $\operatorname{dim} T$ for any killer.) 
Expectably, 5.1 and 5.2 are the easiest to settle. The counterexamples are given by t.c. presentations.

5.1. Let $S$ be generated by $x, y$ subject to $3 x=2 y$. Relative to $X=\{x, y\}$ one finds that $K=\left\{2 k x+3 k y \in Z_{X} ; k \in Z\right\}$; hence $S^{*} \cong C \cong N^{0}$ is free; but $S$ is not. Up to isomorphism, $F(S)=N^{0}, S=\{2,3,4, \ldots\}$.

5.2. Let $S$ be generated by $x, y, z$ subject to $5 x+2 y=3 z$ (there are simpler examples, but this semigroup will be used again). Then $u \in C$ if and only if $u_{x} \geqq 0$ etc. and $5 u_{x}+2 u_{y}=3 u_{z}$. One finds that $B$ consists of $a=3 y+2 z, b=x+2 y+3 z$, $c=2 x+y+4 z, d=3 x+5 z$. Then $S^{*} \cong C$ is not free, since $a+d=b+c$.

5.3. Let $S$ be as above, and $H$ be the pure subgroup of $Z_{B}$ generated by $a+4 b$ $-2 c-3 d ; H$ determines a t.c. congruence $\mathscr{C}$ on $F(S)$. On the other hand, $\alpha(S)$ is generated by $\alpha(x)=b+2 c+3 d, \alpha(y)=3 a+2 b+c, \alpha(z)=2 a+3 b+4 c+5 d$, so that the subgroup $G$ of $Z_{B}$ generated by $\alpha(S)$ consists of all $(3 v+2 w) a+(u+2 v+3 w) b$ $+(2 u+v+3 w) c+(3 u+5 w) d$, where $u, v, w \in Z$. It is immediate to verify that $H \cap G=\{0\}$. Therefore $\varphi: F(S) \rightarrow F(S) / \mathscr{C}=U$ is one-to-one on $\alpha(S)$ but not one-to-one, even though $U$ is t.c. and in fact reduced.

5.4. Let $S$ and $U$ be as before; we may identify $S$ and $\varphi(\alpha(S))$, and then $U$ becomes a canonical killer of $S$. We take $S$ without identity element, so that $U-\{0\}$ is also a canonical killer of $S$. We use the technique described in the proof of 4.10 to show that $T=U-\{0\}$ is in fact a minimal killer of $S$. First one sees that $u \in Z_{B}$ is in $H$ if and only if $u_{b}+2 u_{c}=2 u_{a}+u_{b}+2 u_{d}=2 u_{a}+u_{c}=0$ (we can obtain equations with all coefficients positive, but they would be more complicated). One can then write the system (1) and solve it. One finds that it has only one solution in nonnegative integers, namely the obvious solution $n_{a}^{a}=n_{b}^{b}=n_{c}^{c}=n_{d}^{d}=1$ and all other unknowns equal to zero. It follows that $T$ is a minimal killer.

From the presentation of $S$ it follows that rel $\operatorname{dim} S=1$ and free $\operatorname{dim} S=2$. Similarly, $T$ has a t.c. presentation in terms of $a, b, c, d$ subject to $a+4 b=2 c+3 d$. Hence $T$ is not free, which disposes of 5.4. In fact $\operatorname{rel} \operatorname{dim} T=1$ and free $\operatorname{dim} T=3$, which answers the last two questions.

2. The following problems are unsolved as yet.

5.7. Is the subgroup of $Z_{B}$ generated by $\alpha(S)$ always pure?

5.8. When is $S \cong T^{*}$ for some $T$ ? When is $\sigma$ an isomorphism? (Note: these two problems are equivalent by 3.8$)$.

5.9. Is $\sigma(S)$ always dense in $S^{* *}$ relative to all semigroups?

Added in Proof. 5.8 is not unsolved anymore.

\section{REFERENCES}

1. A. H. Clifford and G. B. Preston, The algebraic theory of semigroups. Vols. 1, 2, Math Surveys, no. 7, Amer. Math. Soc., Providence, R. I., 1961; 1967. MR 24 \#A2627; MR 36 \#1558.

2. P. A. Grillet, On free commutative semigroups, J. Natur. Sci. and Math. 9 (1969), 71-78.

3. J. R. Isbell, Epimorphisms and dominions, Proc. Conf. Categorical Algebra (La Jolla, Calif., 1965), Springer, New York, 1966, pp. 232-246. MR 35 \#105a. 
4. T. J. Head, Homomorphisms of commutative semigroups as tensor maps, J. Natur. Sci. and Math. 7 (1967), 39-49. MR 37 \#6386.

5. D. B. McAlister, A homomorphism theorem for semigroups, J. London Math. Soc. 43 (1968), 355-366. MR 37 \#329.

6. L. Redei, Theorie des endlich erzeugbaren kommutativen Halbgruppen, Hamburger Math. Einzelschriften, Heft 41, Physica-Verlag, Würzburg, 1963. MR 28 \#5130.

Kansas State University,

ManhatTan, Kansas 66502 